\title{
Bone events and evolution of biologic markers in Gaucher disease before and during treatment
}

\author{
Jérôme Stirnemann ${ }^{1,2^{*}}$, Nadia Belmatoug ${ }^{3}$, Corine Vincent ${ }^{2}$, Olivier Fain ${ }^{1}$, Bruno Fantin ${ }^{3}$, France Mentré ${ }^{2}$
}

\begin{abstract}
Introduction: Known biomarkers of Gaucher-disease activity are platelets, chitotriosidase, angiotensin-converting enzyme (ACE), tartrate-resistant acid phosphatase (TRAP) and ferritin. The aim of this study was to retrospectively evaluate the frequency of bone events (BE) and biomarker changes during two periods: diagnosis to first enzymereplacement therapy (ERT) and the latter to the closing date.

Methods: BE of 62 treated patients, among the 73-patient cohort followed at Beaujon Hospital, Clichy, France, were described with Kaplan-Meier curves, and linear-mixed models were used to analyze their biomarker changes and the influence of several covariates (splenectomy, diagnosis year, genotype, age at diagnosis and sex).

Results: BE occurred before (54 events in 21 patients), but also during, ERT (12 events in 10 patients), with respective frequencies (95\% confidence interval) at 10 years of 22.4\% (13.3 to 36.3) and 20.0\% (10.2 to 36.9). Biomarker slope changes before and during ERT differed significantly for platelets $\left(+190 / \mathrm{mm}^{3} /\right.$ year and $7,035 / \mathrm{mm}^{3} /$ year, respectively; $P<0.0001)$ and ferritin $(+4 \%$ and $-14 \% ; P<0.0001)$. High ferritin levels and low platelet counts at ERT onset were significantly associated with BE during ERT ( $P=0.019$ and 0.039 , respectively). Covariates significantly influenced biomarker changes (baseline and/or slope): splenectomy affected platelets (baseline and changes), TRAP changes and chitotriosidase changes; diagnosis date influenced ACE and TRAP baseline values; and genotype influenced chitotriosidase baseline and changes.
\end{abstract}

Conclusions: Platelet counts and ferritin levels and their slope changes at ERT onset seem to predict BE during treatment. Biomarker baseline values and changes are dependent on several covariables.

\section{Introduction}

Gaucher disease (GD), a rare autosomal-recessive disorder with an approximate prevalence of $1 / 75,000$ live births worldwide, is due to the deficiency of a lysosomal enzyme (glucocerebrosidase, glucosylceramidase or $\beta$ glucosidase acid (EC 3.2.1.45)) [1] or, rarely, its activator (saposin C) [2,3]. This lysosomal storage disease is characterized by liver and spleen enlargement, and severe bone complications [1]. Based on the neurological signs, three clinical phenotypes are recognized: type 1 , the classic form, affects $95 \%$ of the patients and is usually defined by the absence of central nervous system impairment; types 2 and 3 are rare and severe, due to neurological involvement [4].

\footnotetext{
* Correspondence: jerome.stirnemann@jvr.aphp.fr

'Médecine Interne, Hôpital Jean-Verdier, Assistance Publique-Hôpitaux de Paris, Université Paris XIII, Referral Center for Lysosomal Diseases (RCLD),

Avenue du 14 juillet, 93140 Bondy Cedex, France

Full list of author information is available at the end of the article
}

Type $1 \mathrm{GD}$ has bone complications that can alter the functional prognosis: abnormal bone deformity, such as widening of the femur metaphysis (Erlenmeyer flask), osteopenia, osteoporosis, lytic lesions and pathological and vertebral compression fractures. Bone infarcts are manifested by acute painful bone crises, and avascular necroses lead to degenerative arthropathy that may require replacement by prosthesis [5]. Thrombopenia and anemia are common. Liver enzymes may be slightly elevated and cholestasis may be present. GD diagnosis is confirmed by the detection of low glucocerebrosidase activity, usually less than $30 \%$ of the normal value in peripheral leukocytes. Genotyping can sometimes provide prognosis information [6].

Enzyme-replacement therapy (ERT), alglucerase then imiglucerase, available since 1991, is the reference treatment. Substrate-reduction therapy (miglustat) has been available since 2002, and is indicated for moderate GD when ERT is unsuitable. These treatments are extremely 
expensive. ERT appears to be ineffective against the onset of neurological disorders in type 2 [4,7]. To our knowledge, no complete analysis of bone complications occurring under ERT is available. Bone complications generally decline after two years of ERT [8]. However, no data are available on the main bone events (BE; avascular necrosis, bone infarcts, pathological fractures) occurring during ERT.

Several biomarkers (chitotriosidase, ferritin, angiotensin-converting enzyme (ACE) and tartrate-resistant acid phosphatase (TRAP)) are elevated during GD evolution [9-16]. Their concentrations rise with disease progression and generally decrease during ERT [17]. At present, it is not possible to make any formal recommendations concerning the use of any specific marker for patient monitoring [18]. Moreover, it is not known if biomarker levels at diagnosis can predict GD prognosis of treated and untreated patients, and which patients will respond, or not, to therapy [19]. Despite the lack of official guidelines, ACE, TRAP and chitotriosidase are used to monitor GD follow-up [20].

Several studies on chronic diseases used biomarker modeling to describe their evolution: human immunodeficiency virus infection [21], Parkinson disease [22] and diabetes mellitus [23]. Concerning GD patients, most published studies are descriptions of small cohorts (median number of patients, 29; range, 18 to 48) $[9,10,17,19,24,25]$ and only one study modeled hemoglobin and platelet levels and splenic volume under ERT [26].

Therefore, this study was undertaken to analyze BE frequencies occurring during the periods before and during ERT in our cohort of GD patients and to model the progression of their biological marker levels or slope changes.

\section{Materials and methods}

\section{Patients and data collected}

The Referral Center for Lysosomal Diseases (RCLD) is specialized in GD follow-up. A designated French national GD registry was developed and is maintained by the RCLD. Although patients are treated and followed in hospitals near their homes, they are registered with the RCLD, which is available to assist their physicians. However, a cohort of patients is followed and treated on-site in the RCLD. All patients with known GD entered in the RCLD registry, followed on-site in the Department of Internal Medicine, Beaujon Hospital (Clichy, France), and receiving ERT were included. Clinical, biological and radiological data were recorded for all patients from diagnosis until 1 May 2007, the closing date. Data were collected retrospectively for two periods: before and during ERT.
Written consent was obtained from each patient. The local Institutional Review Board of Northern Paris Hospitals, Paris-Diderot University, AP-HP (Ethics Committee) reviewed and approved the research project.

A standardized case-report form was used to collect the following information at each visit: initial data (age, sex, history related or unrelated to GD, initial symptoms and their year of onset, test confirming the diagnosis, phenotype, genotype, unknown genetic mutation); clinical information during the first consultation, at diagnosis and throughout follow-up; organomegaly (liver and/ or spleen), usually measured using diagnostic ultrasonography (largest diameter); biological findings initially and throughout follow-up. Bone findings (X-rays, magnetic resonance imaging and, for some patients, scintigraphy and dual-energy X-ray absorptiometry) were recorded during follow-up, with identification of intercurrent events, particularly bone complications.

$\mathrm{BE}$ were defined as clinical events using the bone indications for treatment recommended by the French National Health Authority [27]: avascular necrosis of an epiphysis, bone infarct, pathological and/or vertebral compression fracture(s). Each BE had a clinical manifestation and radiological confirmation. Bone pain alone was not considered a BE without radiological confirmation.

Monitoring of GD-specific ERT and combined therapies (analgesics and bisphosphonates) were noted.

GD diagnosis was confirmed by low glucocerebrosidase activity in leukocytes [28] for all patients. Chitotriosidase activity in plasma samples was determined using the fluorescent substrate 4-methyl umbeliferyl $\beta$ $d-N, N^{\prime}, N^{\prime \prime}$-(MU)-triacylchitotriose [9]; ACE, TRAP, ferritin and other measurements were made in the appropriate local laboratories. Because this study was retrospective, some data were missing, particularly at the beginning of follow-up (during the diagnosis phase). When missing, the baseline value at ERT onset was replaced with the last known value during the two previous years. When the chitotriosidase concentration was undetectable (patients with homozygous chitotriosidasegene deficiency), this biomarker was not retested [29].

\section{Statistical analysis}

All statistical analyses were performed with SAS software (version 9.1; SAS Institute Inc, Cary, North Carolina, USA). The significance level was set at $P<0.05$.

First, we described BE frequency using Kaplan-Meier probability-of- $B E$ curves to determine the time to the first $\mathrm{BE}$ for treated patients between diagnosis and their first treatment (before ERT), and between first ERT and the closing date (during ERT). Only the first BE occurring during each period was considered. Data were 
censored if no BE occurred before the patient started ERT for the first analysis and until the closing date for the second analysis. The LIFETEST procedure was used.

Second, we analyzed the changes of the five GD biomarkers (platelets, chitotriosidase, ferritin, ECA, TRAP) using linear-mixed models for repeated measures with the MIXED procedure. Because of their minimal variations during ERT, hemoglobin levels were not included in this model. The MIXED procedure is a generalization of a standard linear regression, which allows modeling of the parameter changes for each individual over time and takes into account the intrasubject association. Biomarker changes over time could have one of two shapes: either a linear increase or an exponential decrease. For the latter, logarithmic transformation was used in the model. Models of platelet changes used only the counts of nonsplenectomized patients. Two categories were created and analyzed: before and during ERT, regardless of the dose, with analysis of patients receiving full-dose ERT as a subcategory. Before-and-during ERT slopes were compared using the Wald test.

Third, we analyzed the effects of five covariates on BE: splenectomy, diagnosis year (before 1991 or after 1991, the year ERT became available), genotype (N370S/ N370S or others), age at diagnosis (before 15 or after 15 years old) and sex. The impact of each covariate on the time to the first $\mathrm{BE}$ was tested using the log-rank test. A Cox model, used to estimate hazard ratios (HR) and 95\% confidence intervals (CI), was applied to patients before and during ERT. Influence of age at treatment onset on BE occurrence under ERT was tested with a Cox model.

Fourth, the covariates were tested in mixed models. These analyses were only applied to the patients under ERT because of insufficient data on the patients before ERT. Backward selection of the covariates entered into the model was applied to examine associations between a biomarker and the different covariates. For the beforeERT and during-ERT analysis periods, individual baseline and slope values estimated with the linear-mixed models with no covariate for each patient and for each biomarker were extracted. These values were entered into a Cox model, to evaluate the relationship between $\mathrm{BE}$ and biomarker changes using the PHREG procedure, and are expressed as $\mathrm{HR}$ and $95 \% \mathrm{CI}$.

A biomarker effect on BE occurrence before ERT was not analyzed because early information on biomarkers was very sparse.

\section{Results}

\section{Cohort}

Seventy-three patients were followed, between 1933 and 1 May 2007, for a median duration of follow-up of 21 (range, 0 to 67) years after diagnosis. Only 62 patients received ERT with a median total duration of follow-up from diagnosis of 23.5 (range, 2 to 67) years and median duration of follow-up under treatment of 6 (range, 0 to $15)$ years. Only these 62 patients were included in the analysis.

The patients' characteristics at diagnosis are reported in Table 1. This mostly female cohort had a median age of 14 years at diagnosis, but their first symptoms had started at the median age of eight years. Bonemarrow aspiration or biopsy led to GD diagnosis for $32(51 \%)$ patients, and spleen histology was used for $13 \%$. The diagnosis was confirmed for all patients by determining glucocerebrosidase activity. Only one patient died of GD-associated pulmonary hypertension during follow-up.

All but four patients had phenotype $1 \mathrm{GD}$. Twentyeight patients had familial GD affecting siblings for 27 patients and an uncle for one patient. The genotype

Table 1 Description at diagnosis of the 62 Gaucherdisease patients receiving enzyme-replacement therapy

\begin{tabular}{|c|c|}
\hline Baseline characteristic & Value \\
\hline \multicolumn{2}{|l|}{$\overline{S e x, n(\%)}$} \\
\hline Female & $36(58)$ \\
\hline Male & $26(42)$ \\
\hline \multicolumn{2}{|l|}{ Age, years, median (range) } \\
\hline First symptoms & $8(0$ to 37$)$ \\
\hline Diagnosis & $14(1$ to 48$)$ \\
\hline Patients diagnosed before 1991, n (\%) & $47(76 \%)$ \\
\hline Patients $<15$ years old at diagnosis, $n(\%)$ & $34(55 \%)$ \\
\hline First symptoms to diagnosis interval, years, median (range) & $1(0$ to 36$)$ \\
\hline \multicolumn{2}{|l|}{ Test leading to diagnosis, $n(\%)$} \\
\hline Enzyme assay & $7(11)$ \\
\hline Enzyme-gene sequencing & $1(2)$ \\
\hline Myelogram & $26(42)$ \\
\hline Bone-marrow biopsy & $4(6)$ \\
\hline Bone biopsy & $2(3)$ \\
\hline Hepatic biopsy & $2(3)$ \\
\hline Spleen histology & $8(13)$ \\
\hline Other & $1(2)$ \\
\hline Unknown & $11(18)$ \\
\hline \multicolumn{2}{|l|}{ Phenotype, n (\%) } \\
\hline 1 & $58(93)$ \\
\hline 3 & $4(7)$ \\
\hline \multicolumn{2}{|l|}{ Genotype, $n$ (\%) } \\
\hline N370S/N370S & $9(14)$ \\
\hline N370S/L444P & $12(19)$ \\
\hline Other & $27(44)$ \\
\hline Unknown & $14(23)$ \\
\hline \multicolumn{2}{|l|}{ Familial disorder, $n(\%)$} \\
\hline Yes & $28(45)$ \\
\hline No & $23(37)$ \\
\hline Unknown & $11(18)$ \\
\hline
\end{tabular}


was known for more than $90 \%$ of the patients, including nine N370S/N370S, 12 N370S/L444P and two L444P/ L444P.

The first ERT prescribed was alglucerase for 18 (29\%) patients and imiglucerase for 44 (71\%); ERT was started at a median of 14 (range, 0 to 61) years after diagnosis; median age (range) at ERT onset was 31.6 (4.4 to 65.9) years. The respective median ages (range) at treatment onset for patient with and without BE were 29.5 (15.6 to $51.1)$ years and 32 (4.4 to 65.9) years. Cox analysis results showed no influence of age at treatment onset on BE occurrence during ERT (HR = 1.017 (95\% CI: 0.975 to 1.061$), P=0.42$ ). All patients taking alglucerase were switched to imiglucerase in November 1996. For alglucerase or imiglucerase, the initial dose was $120 \mathrm{U} /$ $\mathrm{kg} / \mathrm{month}$ (full dose) for 55 patients, with lower doses for the others: median 90 (range, 30 to 90 ) $\mathrm{U} / \mathrm{kg} /$ month. Four patients switched from imiglucerase to miglustat. Twenty-eight of the 55 patients receiving full doses had their doses lowered after a median of 2.9 (range, 0.1 to 12.2) years of ERT.

Table 2 shows clinical, biological and bone data at different times: diagnosis, ERT onset and closing date. However, median times to ERT onset differed when GD had been diagnosed before 1991 or after 1991 (respectively 18 and 7 years; $P<0.05$ ). Median ages at ERT onset and the closing date were 33.3 and 39.8 years, respectively.

During ERT, clinical abnormality rates decreased (except for neurological involvement) and the biological data improved overall during ERT (increased hemoglobin, leukocyte and platelet levels; decreased chitotriosidase, ACE, TRAP, ferritin and gammaglobulin levels). The number of patients with splenomegaly and/or hepatomegaly tended to decline during ERT, and most bone lesions other than $\mathrm{BE}$ tended to regress (Table 2).

Overall, $21(34 \%)$ patients were splenectomized: 5 before diagnosis, 16 between diagnosis and ERT onset; none were splenectomized after starting ERT. The median age at splenectomy was 18.3 (range, 1.6 to 49.6 ) years.

\section{BE characteristics}

Kaplan-Meier curves of the time to the first BE in the 62 treated patients, between diagnosis and ERT onset (30 years of follow-up), and between the latter and the closing date (15 years of follow-up) are shown in Figure $1 \mathrm{a}$ and $1 \mathrm{~b}$, respectively. Before diagnosis, eight patients had already suffered at least one BE. After diagnosis, but before starting ERT, 21 patients had had at least one $\mathrm{BE}$, for a total of $54 \mathrm{BE}$ and a median of two (range, 1 to 8) BE per patient. Ten patients had at least one BE during ERT for a total of $12 \mathrm{BE}$. The 54 BE before ERT onset were (n (\%)): 28 (52\%) avascular necroses (with 12 prosthetic replacements), 7 (13\%) bone infarcts (with only symptomatic therapy), 12 (22\%) pathologic fractures (5 requiring surgical intervention) and 7 (13\%) vertebral compression fractures (with symptomatic therapy). Moreover, 23 complaints of bone pain were not corroborated by imaging (hence not included in $\mathrm{BE})$. The $12 \mathrm{BE}$ that occurred under ERT were (n (\%)): three (25\%) avascular necroses (none with prosthetic replacement), four (33\%) bone infarcts with clinical bone crises (with only symptomatic therapy) and five (42\%) pathological fractures (none requiring surgery). Twenty-one complaints of simple bone pain without imaging confirmation during ERT were not included in BE. For nine of the $12 \mathrm{BE}$, patients received full-dose ERT (120 U/ kg/month). Only one patient experienced BE during the first year of ERT (pathological fracture), and 5 of the 10 patients experienced BE between Years 1 and 5 of ERT (two pathological fractures, one avascular necrosis and two bone infarcts).

We determined the probability of a $\mathrm{BE}$ occurring by 10 years $(95 \% \mathrm{CI})$ before and during ERT: $22.4 \%$ (13.3\% to $36.3 \%)$ and $20.0 \%$ (10.2\% to $36.9 \%)$, respectively. Respective mean times $(95 \% \mathrm{CI})$ to the first $\mathrm{BE}$ were 27.6 (21.5 to 33.7$)$ and 12.0 (10.7 to 13.3 ) years. For four of the $21(19.0 \%)$ patients with at least one $\mathrm{BE}$ before ERT, the BE occurred before and during ERT, whereas six of the remaining 41 (14.6\%) patients developed BE only during ERT, but had never done so before.

\section{Biomarker evolution before and during ERT}

Results of analyses of biomarker changes using linearmixed models are reported in Table 3. Platelet counts in nonsplenectomized patients were stable before ERT (+190 platelets/year), while chitotriosidase and TRAP decreased slightly, and ferritin and ACE increased slightly. During ERT, platelet counts increased $(+7,035$ platelets/year), while all other biomarkers declined. Slopes before and during ERT differed significantly $(P=$ $0.0001)$ only for platelets and ferritin. For patients given full-dose ERT, platelet counts increased slightly faster, ferritin and TRAP decreased faster, but chitotriosidase and ACE declined more slowly. When only patients with full-dose ERT were analyzed, their biomarker-slope variations were comparable to those of the other patients.

\section{Impact of covariates and biomarkers on developing $\mathrm{BE}$}

Covariate (splenectomy, date of diagnosis, genotype, age at diagnosis and sex) impact on BE before and during ERT was examined. Before ERT, significantly more BE occurred in patients diagnosed with GD after 15 years of age (HR, 2.6 (95\% CI, 1.0 to 6.7); $P=0.048)$, but no 
Table 2 Clinical, biological and imaging characteristics of Gaucher disease precisely known at each time

\begin{tabular}{|c|c|c|c|c|c|c|}
\hline Characteristic & No. & At diagnosis & No. & At ERT onset & No. & At closing date \\
\hline Years since diagnosis, median (range) & & 0 & & $14(0$ to 61$)$ & & 23.5 (2 to 67 ) \\
\hline \multicolumn{7}{|l|}{ Clinical involvement } \\
\hline Pigmentation & 35 & $6 \%$ & 35 & $20 \%$ & 29 & $3 \%$ \\
\hline Asthenia & 45 & $42 \%$ & 52 & $60 \%$ & 53 & $26 \%$ \\
\hline Abdominal pain & 45 & $29 \%$ & 50 & $34 \%$ & 54 & $6 \%$ \\
\hline Chronic bone pain & 1 & $0 \%$ & 52 & $58 \%$ & 55 & $45 \%$ \\
\hline Bone crisis & 38 & $24 \%$ & 45 & $49 \%$ & 51 & $12 \%$ \\
\hline Hemorrhage & 44 & $52 \%$ & 53 & $43 \%$ & 53 & $9 \%$ \\
\hline Lung & 41 & $2 \%$ & 49 & $0 \%$ & 52 & $6 \%$ \\
\hline Neurological & 40 & $5 \%$ & 45 & $7 \%$ & 41 & $15 \%$ \\
\hline Other & 28 & $4 \%$ & 32 & $6 \%$ & 19 & $0 \%$ \\
\hline Splenectomy, $n$ & 62 & 5 & 62 & 21 & 62 & 21 \\
\hline \multicolumn{7}{|l|}{ Organomegaly } \\
\hline Hepatomegaly & 48 & $85 \%$ & 47 & $89 \%$ & 39 & $54 \%$ \\
\hline Liver US (median, range), cm & 23 & 16.5 (13 to 25$)$ & 28 & 19 (13 to 30$)$ & 9 & 14 (11.8 to 19$)$ \\
\hline Splenomegaly & 40 & $100 \%$ & 41 & $95 \%$ & 30 & $67 \%$ \\
\hline Splenic US (median, range), $\mathrm{cm}$ & 34 & 18.75 (9.5 to 30$)$ & 26 & 19.4 (9.5 to 31.5$)$ & 11 & 15.2 (9 to 22 ) \\
\hline \multicolumn{7}{|l|}{ Biological parameter, median (range) } \\
\hline Hemoglobin (g/dL) & 15 & $11.4(7.9$ to 14.1$)$ & 55 & $12(8.3$ to 15.1$)$ & 58 & 13.8 (7.3 to 16.2$)$ \\
\hline Leukocyte $\left(/ \mathrm{mm}^{3}\right)$ & 15 & $4,300(2,070$ to 12,400$)$ & 54 & $4,200(1,180$ to 21,600$)$ & 57 & $6,130(850$ to 11,600$)$ \\
\hline Platelet count $\left(\times 10^{3} / \mathrm{mm}^{3}\right)$ & 57 & 88 (6 to 380$)$ & 53 & 87 (30 to 449$)$ & 58 & $165.5(37$ to 473$)$ \\
\hline Chitotriosidase $(\mathrm{nmol} / \mathrm{mL} / \mathrm{h})^{*}$ & 28 & $9,501$ (70 to 77,500$)$ & 27 & $9,700$ (180 to 77,500$)$ & 53 & 1123 (8 to 14,893 ) \\
\hline TRAP (IU/L) & 23 & 11 (1 to 47$)$ & 15 & 9.6 (1 to 24.5$)$ & 36 & 3.75 (2 to 48 ) \\
\hline ACE (IU/L) & 28 & 259.5 (1 to 650$)$ & 21 & 220 (1 to 650$)$ & 46 & 51 (0.9 to 240) \\
\hline Ferritin (ng/L) & 38 & 682.5 (68 to 3,230$)$ & 28 & 721.5 (120 to 3,230$)$ & 47 & $167(15$ to 1,731$)$ \\
\hline Gammaglobulin (g/L) & 2 & $17.7(16.5$ to 19$)$ & 31 & 15.8 (7.2 to 25$)$ & 47 & 12.7 (6.5 to 23.6$)$ \\
\hline \multicolumn{7}{|l|}{ Imaging of bone disorders } \\
\hline Erlenmeyer flask & 23 & $52 \%$ & 22 & $64 \%$ & 14 & $36 \%$ \\
\hline Osteopenia & 23 & $57 \%$ & 18 & $56 \%$ & 17 & $47 \%$ \\
\hline Cortical & 19 & $32 \%$ & 13 & $23 \%$ & 9 & 0 \\
\hline Lytic lesion & 19 & $26 \%$ & 14 & $21 \%$ & 15 & $33 \%$ \\
\hline Avascular necrosis sequelae & 27 & $37 \%$ & 17 & $29 \%$ & 13 & $23 \%$ \\
\hline Infarct sequelae & 22 & $32 \%$ & 15 & $40 \%$ & 12 & $25 \%$ \\
\hline Fracture sequelae & 18 & $17 \%$ & 11 & 0 & 11 & $27 \%$ \\
\hline Infiltration on MRI & 25 & $80 \%$ & 33 & $91 \%$ & 31 & $81 \%$ \\
\hline${ }^{99 m} \mathrm{Tc}-$ Hyperfixation & 25 & $84 \%$ & 29 & $90 \%$ & 25 & $88 \%$ \\
\hline${ }^{99 m}$ Tc-Hypofixation & 4 & $50 \%$ & 5 & $20 \%$ & 3 & 0 \\
\hline \multicolumn{7}{|l|}{ Bone densitometry, median (range) } \\
\hline T-score neck & 3 & $-2.1(-2.2$ to -1.1$)$ & 10 & $-1(-2.2$ to 1.4$)$ & 22 & $0(-2.8$ to 4.5$)$ \\
\hline T-score lumbar & 13 & $0(-3.1$ to 1$)$ & 10 & $-1.9(-4$ to 0.8$)$ & 22 & $-0.9(-3.6$ to 1.6$)$ \\
\hline Z-score neck & 7 & 8 (-2.03 to 8$)$ & 7 & $-0.7(-2$ to 1.9$)$ & 15 & $-0.3(-2.6$ to 4.4$)$ \\
\hline Z-score lumbar & 2 & $-2.4(-3.1$ to -1.8$)$ & 8 & $-1.3(-3.1$ to 0.5$)$ & 15 & $-0.5(-3$ to 2.1$)$ \\
\hline
\end{tabular}

No. is the number of patients with available information. ACE, angiotensin-converting enzyme; ERT, enzyme-replacement therapy; MRI, magnetic resonance imaging; TRAP, tartrate-resistant acid phosphatase; US, ultrasonography. ${ }^{*}$ In addition, four patients had undetectable chitotriosidase activity (null allele) and are not included for statistical analysis of chitotriosidase.

significant differences were found for the other covariates, and none had an effect during ERT.

Estimated individual slopes of the biomarkers had no significant influence on developing BE before or during ERT but, at diagnosis, ferritin concentration (HR, 1.18 (95\% CI, 1.03 to 1.35 ); $P=0.019$ ) and platelet count
(HR, 0.69 (95\% CI, 0.49 to 0.98); $P=0.039$ ) increased the risk of BE during ERT in this univariate model. Risk of $\mathrm{BE}$ increased with high ferritin levels and low platelet levels.

For patients under ERT, a multivariate regression model including age at diagnosis and ferritin and 


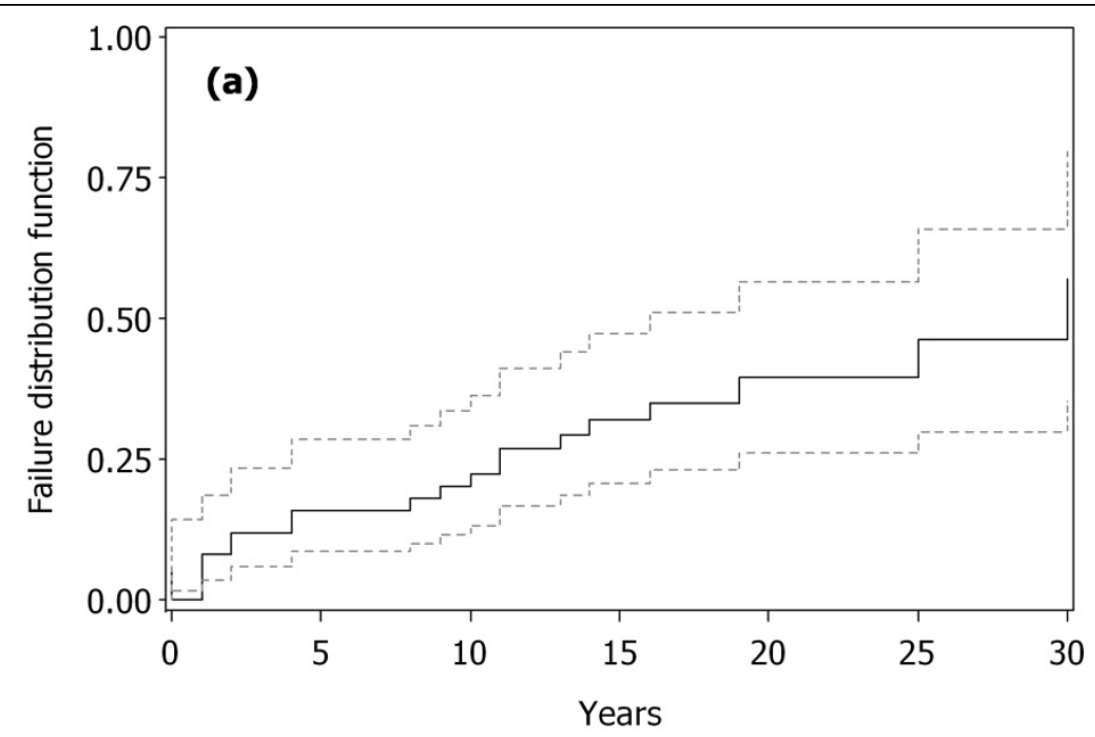

$\begin{array}{rllll}\text { No. at risk } & 62 & 36 & 13 & 5 \\ \text { No. with BE } & 3 & 12 & 18 & 21\end{array}$

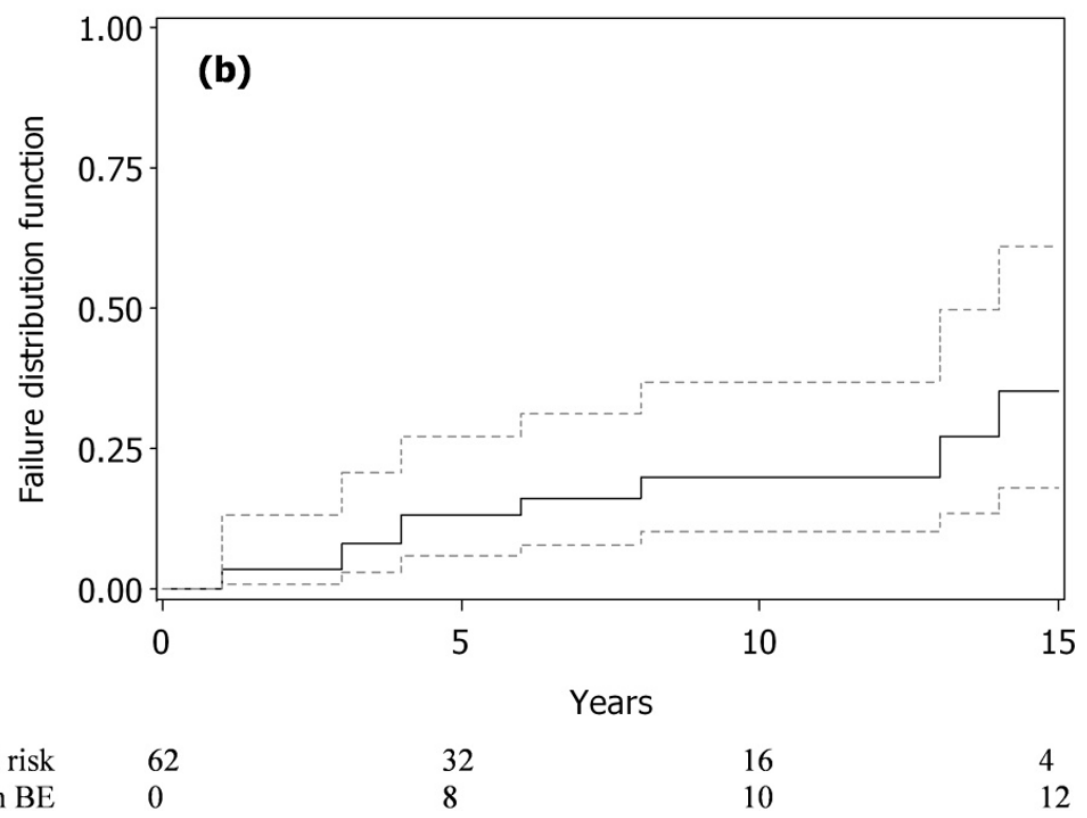

Figure 1 Time until the first bone event (BE) in the 62 Gaucher-disease patients receiving enzyme-replacement therapy (ERT). The dashed grey lines represent the $95 \% \mathrm{Cl}$ of the survival curve. (a) Between diagnosis and first ERT during the first 30 years of follow-up. (b) Between first ERT and closing date (for treated patients) during 15 years of follow-up. No. at risk is the number of patient followed at the indicated time; No. with BE is the number of patients who had a BE. Twenty-one patients had BE before ERT and 10 under ERT during each follow-up period.

platelet levels found only the baseline platelet count $(P=0.032)$ to have a significant impact.

Influence of covariates on biomarker changes during ERT Table 4 reports the effects of covariates on biomarker values during ERT, including the coefficients of variation of interindividual variability for baseline levels and slopes for each biomarker. These coefficients were particularly high for the platelet count, ferritin and ACE slopes. However, only the ferritin and platelet slopes differed significantly between before and during ERT (Table 3). 
Table 3 Changes of the slopes* of the Gaucher-disease biomarkers

\begin{tabular}{|c|c|c|c|c|c|c|c|}
\hline \multirow[b]{2}{*}{ Biomarker } & \multicolumn{6}{|c|}{ Slope of variation (Unit) } & \multirow[t]{2}{*}{$P^{a}$} \\
\hline & No./no. & Before ERT & No./no. & During ERT & No./no. & Full-dose ERT & \\
\hline Platelets (no splenectomy) ${ }^{b}$ & $9 / 70$ & $190 / \mathrm{mm}^{3} / \mathrm{y}$ & $38 / 480$ & $7,035 / \mathrm{mm}^{3} / \mathrm{y}$ & $33 / 220$ & $10,231 / \mathrm{mm}^{3} / \mathrm{y}$ & $<0.0001$ \\
\hline Chitotriosidase & $10 / 23$ & $-11 \% / y$ & $52 / 226$ & $-17 \% / y$ & $37 / 134$ & $-14 \% / y$ & 0.14 \\
\hline TRAP & $7 / 14$ & $-0.7 \% / y$ & $36 / 214$ & $-4 \% / y$ & 24/109 & $-6 \% / y$ & 0.78 \\
\hline ACE & $9 / 33$ & $0.1 \% / y$ & $46 / 263$ & $-5 \% / y$ & $31 / 114$ & $-4 \% / y$ & 0.88 \\
\hline Ferritin & $9 / 45$ & $4 \% / y$ & $47 / 338$ & $-14 \% / y$ & $37 / 166$ & $-16 \% / y$ & $<0.0001$ \\
\hline
\end{tabular}

*Slopes were estimated by linear-mixed models before enzyme-replacement therapy (ERT), during ERT and for the patient subgroup receiving full-dose ERT. ${ }^{a}$ Comparison of slope values before vs during ERT. ${ }^{b}$ Only for nonsplenectomized patients. No./no. indicates number of patients with available data/total number of data available. ACE, angiotensin-converting enzyme; TRAP, tartrate-resistant acid phosphatase.

Figure 2 illustrates the influence of covariates on biomarker changes under ERT. Figure 2a shows the influence of splenectomy on the platelet change. Values at diagnosis and progression slopes differed between splenectomized and nonsplenectomized groups. Splenectomized patients' platelet counts rose slightly under ERT, with no significant slope change, while nonsplenectomized patients' counts increased significantly from baseline under ERT but returned to normal after 6.5 years of treatment. Two covariates affected the chitotriosidase decrease under ERT: splenectomy influenced the slope decline and genotype influenced the baseline level (Figure 2b). Patients with the genotype N370S/N370S had significantly higher baseline levels, which decreased more steeply than those of patients with another genotype. This impact could reflect the fact that, patients with that genotype, which corresponds to one of the less severe forms of GD, started ERT later (median, 23 years) than the other patients (median, 16 years) (nonsiginficant, NS). Chitotriosidase levels decreased, without reaching a normal level over a median of six (range, 0 to 15$)$ years of follow-up.

The ferritin model did not identify any covariate as significantly influencing this marker's progression (Figure $2 c$ ). The mean prediction curve for ferritin levels decreased and became the normal after three years.

Table 4 Baseline levels and slope changes* of the biomarkers during enzyme-replacement therapy: impact of covariates

\begin{tabular}{|c|c|c|c|c|c|c|c|c|}
\hline Biomarker & Covariate(s) & $\mathbf{N}$ & Baseline & $P$ & $\mathrm{CV}^{\mathrm{a}}$ & Slope of \% decrease & $P$ & $\mathrm{CV}^{\mathrm{a}}$ \\
\hline \multicolumn{9}{|l|}{ Platelets } \\
\hline & No splenectomy & 38 & $108,850\left(/ \mathrm{mm}^{3}\right)$ & & $56.5 \%$ & $7035\left(/ \mathrm{mm}^{3} / \mathrm{yr}\right)$ & & $80.7 \%$ \\
\hline & Splenectomy & 20 & $309,380\left(/ \mathrm{mm}^{3}\right)$ & & & $-882\left(/ \mathrm{mm}^{3} / \mathrm{yr}\right)$ & & \\
\hline & Effect of splenectomy & & & $<0.0001$ & & & 0.0007 & \\
\hline \multirow[t]{7}{*}{ Chitotriosidase } & & & & & & & & ND \\
\hline & No splenectomy-N370S & 6 & $14,721 \mathrm{nmol} / \mathrm{mL} / \mathrm{h}$ & & $9.8 \%$ & $-38 \%(/ y r)$ & & \\
\hline & No splenectomy-other genotype & 22 & $3,581 \mathrm{nmol} / \mathrm{mL} / \mathrm{h}$ & & & $-14 \%(/ y r)$ & & \\
\hline & Splenectomy-N370S & 2 & $8,283 \mathrm{nmol} / \mathrm{mL} / \mathrm{h}$ & & & $-27 \%(/ y r)$ & & \\
\hline & Splenectomy-other genotype & 11 & $2,015 \mathrm{nmol} / \mathrm{mL} / \mathrm{h}$ & & & $-2 \%(/ y r)$ & & \\
\hline & Effect of splenectomy & & & 0.19 & & & 0.015 & \\
\hline & Effect of genotype & & & 0.006 & & & 0.018 & \\
\hline Ferritin & & 47 & $382 \mathrm{ng} / \mathrm{L}$ & & $15.4 \%$ & $-14 \%(/ y r)$ & & $83.6 \%$ \\
\hline \multicolumn{9}{|l|}{ ACE } \\
\hline & Diagnosis before 1991 & 35 & 90.3 (IU/L) & & $25.5 \%$ & $-7 \%(/ y r)$ & & $190.1 \%$ \\
\hline & Diagnosis in 1991 and after & 11 & $31.7(\mathrm{IU} / \mathrm{L})$ & & & $4 \%(/ y r)$ & & \\
\hline & Effect of diagnosis data & & & 0.020 & & & 0.055 & \\
\hline \multicolumn{9}{|l|}{ TRAP } \\
\hline & No splenectomy-diagnosis $>15 \mathrm{yr}$ & 10 & $4.2(\mathrm{IU} / \mathrm{L})$ & & $3.6 \%$ & $-4 \%(/ y r)$ & & ND \\
\hline & No splenectomy-diagnosis $15 \mathrm{yr}$ & 13 & $6.6(\mathrm{IU} / \mathrm{L})$ & & & $-9 \%(/ y r)$ & & \\
\hline & Splenectomy-diagnosis $>15 \mathrm{yr}$ & 5 & $3.5(\mathrm{IU} / \mathrm{L})$ & & & $3 \%(/ y r)$ & & \\
\hline & Splenectomy-diagnosis $<15 \mathrm{yr}$ & 8 & $5.4(\mathrm{IU} / \mathrm{L})$ & & & $-2 \%(/ y r)$ & & \\
\hline & Effect of splenectomy & & & 0.32 & & & 0.004 & \\
\hline & Effect of age at diagnosis & & & 0.016 & & & 0.057 & \\
\hline
\end{tabular}

*Estimated with linear-mixed models under treatment. ${ }^{a}$ Coefficient of variation of interpatient variability. ${ }^{b}$ When a log-linear model was used, the evolution is expressed as the percent change of the slope (all markers except platelets). ND, not determined. 


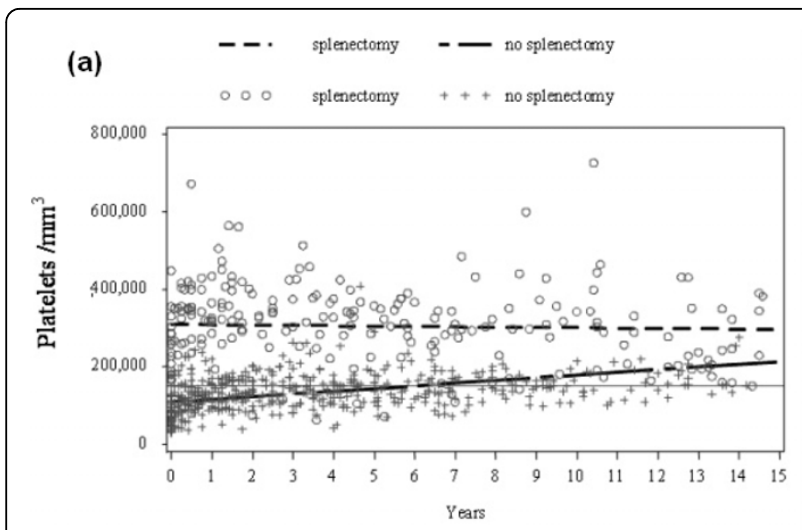

(c)

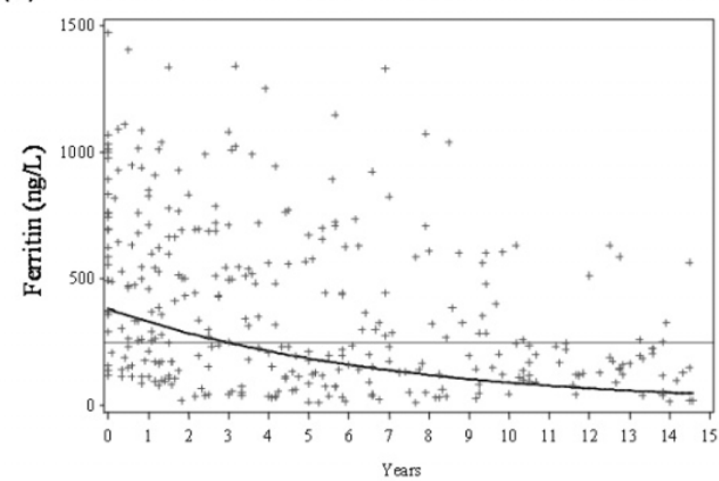

(e)

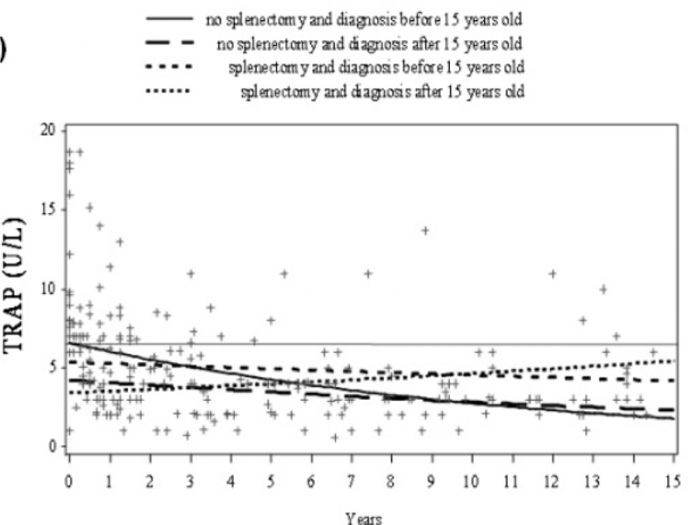

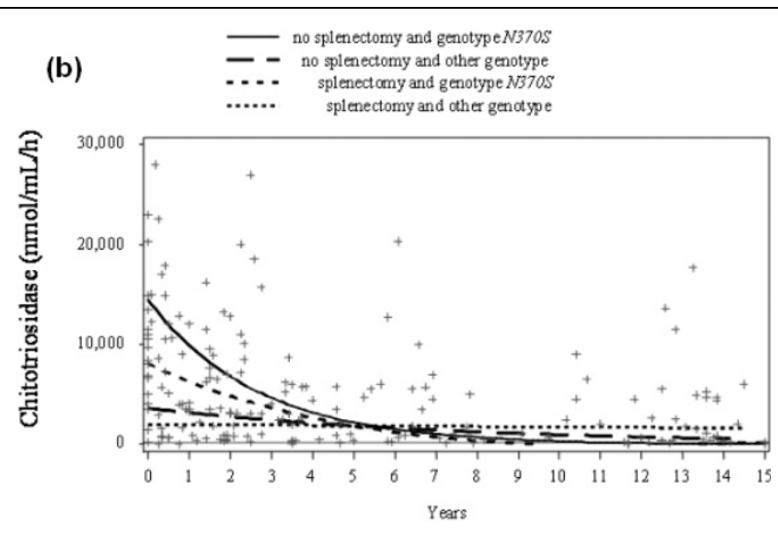

(d)

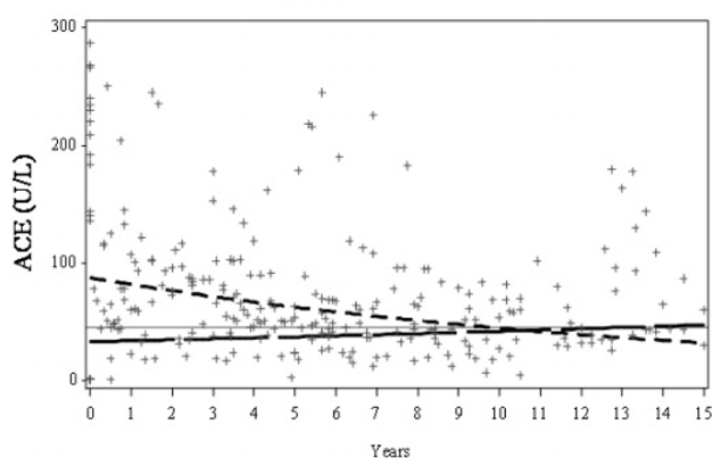

Figure 2 Biomarker changes under enzyme-replacement therapy (ERT): impact of covariates. (a) platelet level, (b) chitotriosidase, (c) ferritin, (d) angiotensin-converting enzyme (ACE), and (e) tartrate-resistant acid phosphatase (TRAP). Different covariates were tested to identify those impacting on biomarker evolution (baseline or variation slope): splenectomy, diagnosis year (before 1991 or after 1991, the year ERT became available), genotype (N370S/N370S or others), age at diagnosis (before 15 or after 15 years old) and sex. Solid grey horizontal lines correspond to the normal biomarker value: platelet level $>150,000 / \mathrm{mm}^{3}$; chitotriosidase $<100 \mathrm{nmol} / \mathrm{mL} / \mathrm{h}$; ferritin $<250 \mathrm{ng} / \mathrm{L}$; ACE $<45 \mathrm{IU} / \mathrm{L}$; TRAP $<7 \mathrm{IU} / \mathrm{L}$. When a covariate had a significant impact on the baseline value or the variation slope, the different curves are shown.

Date of diagnosis significantly affected baseline ACE levels (Figure 2d). Patients diagnosed before 1991 had much higher ACE levels (90.3 versus $31.7 \mathrm{U} / \mathrm{L}$ ), but their progression slopes did not differ significantly.

Splenectomy and age at diagnosis affected TRAP levels, with the former influencing the decrease rate (slope) and the latter the baseline concentration (Figure 2e). TRAP levels in splenectomized patients decreased more slowly and had started higher, but the difference was not significant. Patients diagnosed before the age of 15 years had the highest baseline TRAP levels but their diagnosis-to-ERT interval was 17 years, as opposed to 
11.5 years for those diagnosed after 15 years (NS). Although that difference was not significant, more substrate could have accumulated in the patients who were diagnosed before the age of 15 , because their time preceding ERT onset had been much longer.

Splenectomy eliminates platelet anomalies and displaces lysosomal overload. Nonsplenectomized patients seemed to have higher, but not significantly different, lysosomal biomarker levels at diagnosis compared to splenectomized patients, respectively: chitotriosidase 19,219 versus $2,534 \mathrm{nmol} / \mathrm{mL} / \mathrm{h}$ (NS); TRAP 12.1 versus 8.0 (NS). In contrast, macrophage-biomarker levels were higher in splenectomized than nonsplenectomized patients, respectively: ferritin 1,301 versus $634 \mathrm{ng} / \mathrm{L}(P=$ 0.06); ACE 251 versus 202 IU/L (NS). Generally, for splenectomized patients, when the starting value was lower, the slope was less steep.

Some covariates were significantly associated: patients diagnosed before 1991 were more often male $(P=0.04)$ and had more frequently been splenectomized $(P=$ $0.03)$.

\section{Discussion}

Probability curves of time to BE (Figure 1) before and during ERT for GD patients showed that bone complications could occur without but also under ERT (about $20 \%$ at 10 years). According to the literature, bone crises almost disappeared after two years of ERT $[8,30]$, but the risk of avascular necrosis was estimated to be 13.8/ 1,000 person-years under ERT in a recent publication [31]. Our findings demonstrated that BE can arise even after many years of ERT, specifically avascular necrosis (three patients) and bone infarcts (four patients). However, no patient had prosthetic replacement after ERT compared to 12 before. BE after ERT seemed to be less serious, as no surgery was required. In addition, simple bone pain occurring under ERT without being confirmed by imaging (21 events) was not considered a BE. Hence, ERT does not eliminate all bone symptoms. Nine BE were documented under full-dose ERT (120 U/ $\mathrm{kg} /$ month of alglucerase or imiglucerase). We tried to determine the impact of diagnosis year (before or after 1991) on BE occurrence and biomarker evolution: no effect was found on BE occurrence $(P=0.11$ and 0.42 , respectively, for before and after ERT). Although this covariate significantly affected only baseline ACE levels (see Figure 2d), it had no influence on the other biomarkers, for example, platelets and ferritin. No impact of the date of diagnosis on baseline ferritin value or platelet count was found, as shown in Figure 2a, c and in Table 4.

We hypothesize that bone infiltration accumulating over the years before starting ERT could explain bone complications despite treatment. Unfortunately, our analysis does not allow us to confirm or refute that postulate. In a recent article, the risk of avascular necrosis while on ERT seemed lower for patients who had begun treatment within two years of diagnosis compared to those who started it after more than and equal to two years [31]. Moreover, patients were certainly more closely monitored during than before ERT and it is possible that some BE before ERT might not have been diagnosed. A largest study with systematic bone-density data will be considered to compare the bone densitometries of patients with pathological fractures versus those of patients without such fractures during evolution.

Because the platelet level did not rise in splenectomized patients on ERT, it can be concluded that hypersplenism is the main cause of thrombopenia. However, 14 (61\%) out of 21 splenectomized patients, had at least one platelet count $<150,000 / \mathrm{mm}^{3}$ after splenectomy. Therefore, bone-marrow insufficiency seems to explain part of the thrombopenia observed over time.

The effect of individual estimated-biomarker values at diagnosis or their slope on BE occurrence under ERT was significant for baseline platelet and ferritin levels in our univariate model, with high ferritin and low platelets at ERT onset being significantly associated with BE during ERT, but only the baseline platelet count was retained in a multivariate model. Nor did the estimated individual slopes of the biomarkers have a significant impact on BE. However, our modeling method seemed to be able to identify predictive biomarkers. Effects of others biomarkers was not significant in our analyses but platelet and ferritin data were available for more patients at diagnosis (57 and 38, respectively) compared to the others $(28,23$ and 28, for chitotriosidase, TRAP and ACE, respectively), which could have decreased the power of statistical analysis and partially explain these observations. According to the literature, no biomarkers were able to predict $\mathrm{BE}$ occurrence and, other than platelets, ferritin seemed to be the only biomarker affecting BE [19]. The only study using a mixed model for GD [26] found dose-response relationships for ERT, but had not considered biomarkers (chitotriosidase, TRAP, ferritin, ACE), taking into account only the main hematological (hemoglobin and platelets) and visceral manifestations; no prediction of BE occurrence was proposed.

Interpatient biomarker changes and therapeutic responses varied widely (Table 3 ). TRAP baseline was higher for the group diagnosed before 15 years than those diagnosed later, which seems to support that the TRAP level could differ as a function of age [32]. The respective median ages for the groups of patients diagnosed before and after 15 years were 21 and 40 years, which partially explains these findings. The ACE level was affected by the year of diagnosis, with patients 
diagnosed after 1991 having lower baseline levels than those diagnosed before 1991. ERT availability since 1991 could have limited the ACE rise. For some biomarkers, not all patients had the same evolution under ERT: for example, platelet counts rose in $97 \%$ of treated patients, while chitotriosidase decreased in $100 \%$ and ferritin in $96 \%$, but ACE levels in only $78 \%$.

\section{Conclusions}

BE can occur in GD even after many years of ERT. Initial ferritin and platelet levels seemed to be able to predict BE occurrence during ERT. To achieve our final objective, to predict $\mathrm{BE}$ based on initial biomarker values and their evolution, large cohort studies are needed.

\section{Abbreviations \\ ACE: angiotensin converting enzyme; BE: bone events; Cl: confidence interval; ERT: enzyme replacement therapy; GD: Gaucher disease; HR: hazard ratio; NS: nonsignificant; RCLD: Referral Center for Lysosomal Diseases; TRAP. tartrate-resistant acid phosphatase.}

\section{Acknowledgements}

We thank Janet Jacobson for editorial assistance. A grant from Genzyme France funded, in part, the salaries of a statistician and a clinical research associate who participated in this study. Beaujon Hospital received a grant from Shire which funded Janet Jacobson. Genzyme and Shire played no role in designing the study; collecting, analyzing and interpreting the data; writing the paper; or the decision to submit the manuscript for publication.

\section{Author details}

${ }^{1}$ Médecine Interne, Hôpital Jean-Verdier, Assistance Publique-Hôpitaux de Paris, Université Paris XIII, Referral Center for Lysosomal Diseases (RCLD), Avenue du 14 juillet, 93140 Bondy Cedex, France. ${ }^{2}$ INSERM, UMR 738, Université Paris-Diderot, Hôpital Bichat, 46, rue Henri-Huchard, 75877 Paris Cedex 18, France. ${ }^{3}$ Médecine Interne, Hôpital Beaujon, Assistance PubliqueHôpitaux de Paris, Université Paris VII, Referral Center for Lysosomal Diseases (RCLD), 100, boulevard du Général Leclerc, 92110 Clichy Cedex, France.

\section{Authors' contributions}

FM, JS, CV, NB, BF and OF designed the research protocol. JS, NB, BF and OF were involved in treating patients and collecting data. JS, CV and FM controlled the accuracy of collected data and conducted the statistical analyses. JS, FM and CV wrote the draft of the paper, which was then corrected and approved by all authors.

\section{Competing interests}

Paris-Diderot University received a grant from Genzyme France. JS and NB were reimbursed for congress expenses by Genzyme. Beaujon Hospital received a grant from Shire.

Received: 19 April 2010 Revised: 21 July 2010 Accepted: 9 August 2010 Published: 9 August 2010

\section{References}

1. Brady RO, Kanfer JN, Shapiro D: Metabolism of glucocerebrosides. II. Evidence of an enzymatic deficiency in Gaucher's disease. Biochem Biophys Res Commun 1965, 18:221-225.

2. Christomanou H, Aignesberger A, Linke RP: Immunochemical characterization of two activator proteins stimulating enzymic sphingomyelin degradation in vitro. Absence of one of them in a human Gaucher disease variant. Biol Chem Hoppe Seyler 1986, 367:879-890

3. Qi X, Grabowski GA: Molecular and cell biology of acid beta-glucosidase and prosaposin. Prog Nucleic Acid Res Mol Biol 2001, 66:203-239.
4. Mignot C, Doummar D, Maire I, de Villemeur TB: Type 2 Gaucher disease: 15 new cases and review of the literature. Brain Dev 2006, 28:39-48.

5. Beutler E: Gaucher disease. Arch Intern Med 1999, 159:881-882.

6. Grabowski GA: Recent clinical progress in Gaucher disease. Curr Opin Pediatr 2005, 17:519-524.

7. Erikson A, Bembi B, Schiffmann R: Neuronopathic forms of Gaucher's disease. Baillieres Clin Haematol 1997, 10:711-723.

8. Sims KB, Pastores GM, Weinreb NJ, Barranger J, Rosenbloom BE, Packman S, Kaplan P, Mankin H, Xavier R, Angell J, Fitzpatrick MA, Rosenthal D: Improvement of bone disease by imiglucerase (Cerezyme) therapy in patients with skeletal manifestations of type 1 Gaucher disease: results of a 48-month longitudinal cohort study. Clin Genet 2008, 73:430-440.

9. Hollak CE, van Weely S, van Oers MH, Aerts JM: Marked elevation of plasma chitotriosidase activity. A novel hallmark of Gaucher disease. J Clin Invest 1994, 93:1288-1292.

10. Deegan PB, Moran MT, McFarlane I, Schofield JP, Boot RG, Aerts JM Cox TM: Clinical evaluation of chemokine and enzymatic biomarkers of Gaucher disease. Blood Cells Mol Dis 2005, 35:259-267.

11. Lieberman J, Beutler E: Elevation of serum angiotensin-converting enzyme in Gaucher's disease. N Engl J Med 1976, 294:1442-1444.

12. Zimran A, Kay A, Gelbart T, Garver P, Thurston D, Saven A, Beutler E: Gaucher disease. Clinical, laboratory, radiologic, and genetic features of 53 patients. Medicine (Baltimore) 1992, 71:337-353.

13. Beutler E: Gaucher disease. Blood Rev 1988, 2:59-70.

14. Morgan MA, Hoffbrand AV, Laulicht M, Luck W, Knowles S: Serum ferritin concentration in Gaucher's disease. Br Med J (Clin Res Ed) 1983, 286:1864.

15. Tuchman LR, Suna $H$, Carr JJ: Elevation of serum acid phosphatase in Gaucher's disease. J Mt Sinai Hosp N Y 1956, 23:227-229.

16. Troy K, Cuttner J, Reilly M, Grabowski G, Desnick R: Tartrate-resistant acid phosphatase staining of monocytes in Gaucher disease. Am J Hematol 1985, 19:237-244.

17. Cabrera-Salazar MA, O'Rourke E, Henderson N, Wessel H, Barranger JA: Correlation of surrogate markers of Gaucher disease. Implications for long-term follow up of enzyme replacement therapy. Clin Chim Acta 2004, 344:101-107.

18. Aerts JM, van Breemen MJ, Bussink AP, Ghauharali K, Sprenger R, Boot RG, Groener JE, Hollak CE, Maas M, Smit S, Hoefsloot HC, Smilde AK, Vissers JP, de Jong S, Speijer D, de Koster CG: Biomarkers for lysosomal storage disorders: identification and application as exemplified by chitotriosidase in Gaucher disease. Acta Paediatr Supp/ 2008, 97:7-14.

19. Poll LW, Koch JA, Willers R, Aerts H, Scherer A, Haussinger D, Modder U, vom Dahl S: Correlation of bone marrow response with hematological, biochemical, and visceral responses to enzyme replacement therapy of nonneuronopathic (type 1) Gaucher disease in 30 adult patients. Blood Cells Mol Dis 2002, 28:209-220.

20. Weinreb NJ, Aggio MC, Andersson HC, Andria G, Charrow J, Clarke JT, Erikson A, Giraldo P, Goldblatt J, Hollak C, Ida H, Kaplan P, Kolodny EH, Mistry P, Pastores GM, Pires R, Prakash-Cheng A, Rosenbloom BE, Scott CR, Sobreira E, Tylki-Szymanska A, Vellodi A, vom Dahl S, Wappner RS, Zimran A: Gaucher disease type 1: revised recommendations on evaluations and monitoring for adult patients. Semin Hematol 2004, 41(Suppl 5):15-22.

21. Bonnet $F$, Thiebaut $R$, Chene $G$, Neau D, Pellegrin JL, Mercié $P$, Beylot J, Dabis F, Salamon R, Morlat P: Determinants of clinical progression in antiretroviral-naive HIV-infected patients starting highly active antiretroviral therapy. Aquitaine Cohort, France, 1996-2002. HIV Med 2005, 6:198-205.

22. Post TM, Freijer Jl, DeJongh J, Danhof M: Disease system analysis: basic disease progression models in degenerative disease. Pharm Res 2005, 22:1038-1049.

23. de Winter W, de Jongh J, Post T, Ploeger B, Urquhart R, Moules I, Eckland D, Danhof M: A mechanism-based disease progression model for comparison of long-term effects of pioglitazone, metformin and gliclazide on disease processes underlying type 2 diabetes mellitus. $J$ Pharmacokinet Pharmacodyn 2006, 33:313-343.

24. Vellodi A, Foo Y, Cole TJ: Evaluation of three biochemical markers in the monitoring of Gaucher disease. J Inherit Metab Dis 2005, 28:585-592.

25. Czartoryska B, Tylki-Szymanska A, Gorska D: Serum chitotriosidase activity in Gaucher patients on enzyme replacement therapy (ERT). Clin Biochem 1998, 31:417-420. 
26. Grabowski GA, Kacena K, Cole JA, Hollak CE, Zhang L, Yee J, Mistry PK Zimran A, Charrow J, vom Dahl S: Dose-response relationships for enzyme replacement therapy with imiglucerase/alglucerase in patients with Gaucher disease type 1. Genet Med 2009, 11:92-100.

27. National Diagnosis and Treatment Protocol for Gaucher Disease. [http:// www.has-sante.fr/portail/jcms/c_644042/gaucher-disease-nationa-diagnosisand-treatment-protocol]

28. Daniels LB, Glew RH: Beta-glucosidase assays in the diagnosis of Gaucher's disease. Clin Chem 1982, 28:569-577.

29. Aerts JM, Hollak CE: Plasma and metabolic abnormalities in Gaucher's disease. Baillieres Clin Haematol 1997, 10:691-709.

30. Charrow J, Dulisse B, Grabowski GA, Weinreb NJ: The effect of enzyme replacement therapy on bone crisis and bone pain in patients with type 1 Gaucher disease. Clin Genet 2007, 71:205-211.

31. Mistry PK, Deegan P, Vellodi A, Cole JA, Yeh M, Weinreb NJ: Timing of initiation of enzyme replacement therapy after diagnosis of type 1 Gaucher disease: effect on incidence of avascular necrosis. Br J Haematol 2009, 147:561-570.

32. Magalhaes J, Pinto R, Lemos M, Sa Miranda MC, Poenaru L: Age dependency of serum acid phosphatase in controls and Gaucher patients. Enzyme 1984, 32:95-99.

doi:10.1186/ar3111

Cite this article as: Stirnemann et al:: Bone events and evolution of biologic markers in Gaucher disease before and during treatment. Arthritis Research \& Therapy 2010 12:R156.

\section{Submit your next manuscript to BioMed Central} and take full advantage of:

- Convenient online submission

- Thorough peer review

- No space constraints or color figure charges

- Immediate publication on acceptance

- Inclusion in PubMed, CAS, Scopus and Google Scholar

- Research which is freely available for redistribution

Submit your manuscript at www.biomedcentral.com/submit 